\title{
Calidad instrumental de la carne de cabrito lechal. Revisión bibliográfica
}

\author{
Guillermo Ripoll1,2,*, María Jesús Alcalde ${ }^{3}$ y Begoña Panea ${ }^{1,2}$ \\ 1 Instituto Agroalimentario de Aragón - IA2 - (CITA-Universidad de Zaragoza). Calle Miguel Servet, \\ 177. 50013 Zaragoza, España \\ 2 Centro de Investigación y Tecnología Agroalimentaria de Aragón. Avda. Montañana, 930. 50059 \\ Zaragoza, España \\ 3 Universidad de Sevilla. Ctra. Utrera, 41013 Sevilla, España
}

\section{Resumen}

El ganado caprino es de gran importancia en España, tanto por censo como por su repercusión en la Producción Final Ganadera. Esta revisión bibliográfica se ha realizado de manera sistemática con los estudios encontrados en revistas científicas sobre cabrito lechal con un peso vivo de menos de $13 \mathrm{~kg}$ y alimentado exclusivamente con leche. El peso de la canal de estos cabritos se distribuye en dos grandes grupos, con pesos por encima y por debajo de $5 \mathrm{~kg}$. A ambos pesos de canal, la carne de cabrito lechal se confirma como una fuente de proteína baja en grasa. No se puede concluir que exista una relación lineal del sistema de lactancia o del peso canal con el pH. La carne de cabritos alimentados con leche natural tiene un índice de amarillo más alto que los de la carne de cabritos alimentados con lactoreemplazantes. No se apreció una influencia marcada del peso de la canal ni del sistema de lactación en la dureza, que tuvo un valor medio de $35 \mathrm{~N}$. En líneas generales, la carne de canales de más de $5 \mathrm{~kg}$ tuvo menor porcentaje de ácidos grasos saturados y mayor de insaturados, y una ratio n-6/n-3 mayor que la de carne de canales de menos de $5 \mathrm{~kg}$. Hay muy poca información científica sobre la calidad de carne instrumental de los cabritos, por lo que existe un amplio campo de investigación para cubrir la laguna de conocimiento encontrada.

Palabras clave: $\mathrm{pH}$, cabra, revisión, caprino, canal, calidad de carne.

Instrumental quality of suckling kid meat. A review

\section{Abstract}

Goat production is quite important in Spain having high census and economic impact. This review has been performed systematically with studies about suckling kids with slaughter weight lower than $13 \mathrm{~kg}$ and fed exclusively milk. The carcass weight of kids were distributed into two groups, above and below of $5 \mathrm{~kg}$. Kid meat appears as a useful protein source with low fat content. It was found any linear relationship between rearing system or carcass weight with $\mathrm{pH}$. Meat of kids fed maternal milk had greater yellowness index than those fed milk replacers. Toughness was not affected by carcass weight or rearing system and the average value was $35 \mathrm{~N}$. In general, meat of carcasses heavier than $5 \mathrm{~kg}$ had less

* Autor para correspondencia: gripoll@aragon.es

Cita del artículo: Ripoll G, Alcalde MJ, Panea B (2021). Calidad instrumental de la carne de cabrito lechal. Revisión bibliográfica. ITEA-Información Técnica Económica Agraria 117(2): 145-161. https://doi.org/10.12706/itea.2020.016 
saturated and more unsaturated fatty acids, as well a higher n-6/n-3 ratio than meat of carcasses with less than $5 \mathrm{~kg}$. There is few scientific information on the instrumental quality of suckling kid meat. Therefore, there is a wide field of research to cover the absence of knowledge in this topic.

Keywords: $\mathrm{pH}$, goat, review, caprine, carcass, meat quality.

\section{Introducción}

España es el segundo país productor de ganado caprino de la Unión Europea con 2.764.000 cabezas, solo superada por Grecia con 3.625.000 cabezas (Eurostat, 2019). EI censo de ganado descendió paulatinamente entre los años 2008 y 2012 debido a la desaparición de casi 3.500 explotaciones, para luego remontar y alcanzar el máximo de 3.088.582 cabezas en 2017. Este cierre de explotaciones se debió en parte a la subida de precios de los insumos que obligó en 2011 al sacrificio de rebaños lecheros por la falta de rentabilidad de los mismos (SEOC, 2013). La desaparición de parte de los rebaños lecheros influyó también en el tamaño de las explotaciones, que pasó de 41 cabezas por explotación de media en 2017, a un mínimo de 35 en 2014 para volver a recuperarse en 2018 (Eurostat, 2019; MAPA, 2019a). La producción cárnica de ovino y caprino representó en 2017 el 2,5 \% de la Producción Final Agraria nacional y el 6,6 \% de la Producción Final Ganadera, mientras que la producción de leche de cabra supuso un 1,6 \% de la Producción Final Ganadera (MAPA, 2019a). En 2018, España tuvo una producción de 461.400 toneladas de leche de cabra (MAPA, 2019a), aproximadamente una quinta parte de la producción de leche de cabra de la UE. De la producción que se transforma en España, el 89 \% pasó a la industria para la elaboración de quesos y productos lácteos y el resto se destinó a la elaboración de quesos artesanales en la propia explotación (MAPA, 2019a). Además de la gran importancia de la producción de leche de cabra, el cabrito también supone una producción importante del sec- tor caprino, suponiendo un ingreso del $20 \%$ de los ingresos totales por cabra de la explotación lechera (Castel et al., 2012).

En el año 2017, España produjo el $13 \%$ del total de la carne de caprino de la Unión Europea (FAO, 2019). En el año 2018 se sacrificaron en España 1.371.026 animales de la especie caprina (MAPA, 2019a), de los cuales el $80 \%$ fueron de la categoría cabrito lechal. Esta preferencia a sacrificar el cabrito lechal de bajo peso en oposición al chivo está relacionada con el paso de las explotaciones mixtas a lecheras. Se pasó de sacrificar un $65 \%$ de cabrito y un $20 \%$ de chivo en 1992 a sacrificar el $80 \%$ de los animales como cabrito lechal y el $5 \%$ como chivos en el año 2011. Según datos del MAPA (2019a), en España existe un gradiente descendiente del número de cabezas de ganado caprino que va de Sur a Norte. En Andalucía se mantiene más de un tercio del censo español (37,8 \%), seguido de Extremadura, Castilla La Mancha y Murcia que aúnan un $34,8 \%$ del censo. Finalmente, el resto de España acumula el tercio restante del censo caprino. Las Comunidades Autónomas con mayor número de cabritos lechales sacrificados fueron Andalucía (33,8 \%), las Islas Canarias (13,5 \%), Cataluña (11,9\%) y Murcia (10,8 \%). Las Comunidades Autónomas del norte de España, como Galicia, Principado de Asturias, Cantabria, País Vasco y Navarra tuvieron una producción de cabrito lechal casi testimonial de alrededor del $1 \%$ del total de España (MAPA, 2019a). Comparados con los datos de sacrificio de lechales en 2015, estos valores son muy constantes para cada Comunidad Autónoma, pero hay un cambio notable en Extremadura, que en 2015 sacrificó el $6 \%$ de cabritos lechales mien- 
tras que en 2018 sacrificó solo el 1,5\%. Comparando la distribución de los censos ganado caprino en vivo y la distribución de las cabezas de cabrito lechal sacrificadas se puede observar que existen ciertos desequilibrios. Por ejemplo, Extremadura y Castilla La Mancha tienen porcentajes de censo de animales vivos mucho mayores que los de sacrificios, mientras que, por el contrario, en Castilla y León, Canarias y Cataluña, el porcentaje de sacrificio de cabrito lechal es superior al que le correspondería. Finalmente, los sacrificios de cabrito lechal en Andalucía y Murcia sí son coherentes con el censo de animales vivos. Estos desequilibrios entre las zonas de cría de los animales y las zonas de sacrificio, comercialización y consumo denotan graves problemas estructurales en la estacionalidad de la producción y el modelo de comercialización (Panea et al., 2010) que no acaban de resolverse. De igual manera sucede con la leche; Andalucía, produce más del $40 \%$ de la leche de cabra española y, sin embargo, más del $80 \%$ de esta leche es transformada fuera de la región, ya sea en el resto de España o incluso en otros países europeos (Castel et al., 2012). Este traslado de animales y de leche hace que el valor añadido y el beneficio económico se quede en la zona de destino y no en la de origen, por lo que no repercute directamente en el productor, que puede optar por abandonar la actividad (Panea et al., 2008). A pesar de la importancia del ganado caprino en la Producción Final Agraria y Ganadera, estos y otros problemas estructurales como pueden ser la despoblación en el medio rural y la falta de rentabilidad, sitúan a 19 de las 22 razas autóctonas españolas al borde de la extinción (MAPA, 2019a).

En España podemos encontrar tres tipos comerciales de animales caprinos destinados a la producción cárnica (Alcalde et al., 2010): 1) el cabrito lechal, alimentado principalmente con leche y sacrificado al mes o mes y medio de vida, con un peso vivo que puede oscilar entre $7 \mathrm{~kg}$ y $11 \mathrm{~kg}$. De estos animales se con- siguen canales que van desde los $4 \mathrm{~kg}$ hasta los $7 \mathrm{~kg}$, aproximadamente. 2) el chivo, animal alimentado con leche materna y los recursos obtenidos del pastoreo con su madre. Estos animales se sacrifican con 4 o 5 meses de edad y con $20 \mathrm{~kg}$ a $30 \mathrm{~kg}$ de peso vivo y es un tipo comercial menos demandado. 3) el caprino mayor, procedente del desvieje de cabras de leche y otros animales adultos y que es de poca importancia en el mercado. Según la guía de etiquetado facultativo (BOE, 2011), para animales de edad menor a los 5 meses, las canales de cabrito se clasificarían en lechal ligero, para canales menores o iguales a $5 \mathrm{~kg}$; lechal, para canales entre $5 \mathrm{~kg}$ y $7 \mathrm{~kg}$; y recental para canales de peso superior a $7 \mathrm{~kg}$ e inferior o igual a $10 \mathrm{~kg}$. El peso medio de la canal de cabrito lechal en España fue de $5,1 \mathrm{~kg}$, aunque hay variaciones por Comunidades Autónomas. Por una parte, las Comunidades Autónomas del norte de España, como Galicia, Principado de Asturias, Cantabria, País Vasco y Navarra producen canales con un peso mayor de $6 \mathrm{~kg}$, mientras que Andalucía, las Islas Canarias, Cataluña y La Rioja produjeron canales con un peso medio por debajo de $5 \mathrm{~kg}$ (MAPA, 2019b). Hay un gradiente creciente del peso de la canal desde el sureste de la península hacia el noroeste. Se puede observar cómo las zonas con poca producción de cabrito lechal sacrifican a los animales con pesos de canal mayores y viceversa.

A pesar de la importancia en la producción, se observa un constante descenso del consumo, observado desde el final de los años 80 (Chamorro et al., 2012), que se va confirmando en la última década (MAPA, 2019a). En las estadísticas oficiales aparecen los datos de ovino y caprino agrupados hasta el año 2015 y es difícil valorar el consumo de carne caprina, pero algunas estimaciones dan una cifra menor a los $300 \mathrm{~g}$ de carne por habitante y año (Alcalde et al., 2010). A partir del año 2016 el consumo per cápita de carne de caprino fue de $160 \mathrm{~g}$ y en 2018 de $130 \mathrm{~g}$, pro- 
bablemente por la sustitución de la carne de caprino por otras de menor precio (MartínCerdeño, 2018). Además de la producción para el consumo interno, España es un gran exportador a países de la Unión Europea como Francia, Reino Unido o Portugal, y a terceros países (MAPA, 2019a).

Existe mucha bibliografía sobre la calidad de la carne en general. Sin embargo, la mayoría de los libros académicos que versan sobre ciencia de la carne (Forrest et al., 1979; Price y Schweighet, 1994; Lawrie, 1998; Warris, 2000) adolecen de información sobre la especie caprina, y es inexistente en el caso de cabrito lechal ligero o muy ligero. Tampoco se han encontrado revisiones bibliográficas sobre este tipo de carne. Encontramos pues, una laguna de conocimiento, que esta revisión persigue ayudar a cubrir. Por este motivo, y con el objetivo de conocer y valorizar la calidad instrumental de la carne de cabrito lechal, se realizó una revisión sistemática de los artículos publicados en revistas científicas internacionales.

\section{Metodología}

Tras una búsqueda bibliográfica en la base de datos Scopus (https://www.scopus.com/) con la cadena "kid AND suckling AND meat" donde se encontraron 126 documentos, se procedió a descartar aquellos artículos científicos en los que se estudiaron cabritos con peso vivo superior a $13 \mathrm{~kg}$ y donde el cabrito explícitamente ingiriera otro alimento distinto de leche, o que al mencionar el uso de sistemas extensivos o expresiones como "los cabritos permanecieron con las madres" hacen pensar que no cumple con los requisitos establecidos de alimentación de los cabritos exclusivamente con leche. También se utilizaron los datos del longissimus thoracis et lumborum descartando los de otros músculos. Así pues, la base final de artículos para la revisión consta de 24 artículos (Complemento 1), de los cuales 17 son de autores españoles, 5 de autores italianos, uno de Grecia y otro de Turquía.

Por otra parte, en función del objetivo de cada uno de los estudios, también se encontraron lotes de animales que cumplieron los requisitos mencionados y otros que no. En algunos artículos se compararon cabritos con corderos, y en otros se compararon sistemas de manejo en los que solo algún lote se alimentó de leche exclusivamente, y otros lotes fueron suplementados. Después de eliminar estos grupos de animales, se utilizaron datos de 82 lotes distintos de animales. Sin embargo, no todos los artículos aportaron información completa sobre el peso vivo al sacrificio, peso de la canal caliente o edad. Con los estudios que aportaron datos de rendimiento canal y las pérdidas de oreo, se calcularon las medias del rendimiento canal $(53,60 \%)$ y las pérdidas de oreo $(3,04 \%)$. Se asumieron estos valores y con ellos se calcularon los pesos vivos y de la canal caliente en los artículos incompletos. Igualmente, se usó el valor medio de las pérdidas de oreo para homogeneizar los pesos de la canal que en unos estudios se dan como canal caliente y en otros como canal fría. En el caso del artículo de Caputi-Jambrenghi et al. (2010), que no aporta datos ni de peso vivo ni de canal fría o caliente, sino solo la edad de sacrificio, se tomó como válido el dato de peso vivo de machos de la misma raza y edad (Marsico et al., 1993). De forma similar, el peso vivo de los cabritos del artículo de Sanz-Sampelayo et al. (2006) se calculó a partir de la edad y los datos aportados por Peña et al. (1985) para la misma raza.

\section{Discusión}

\section{Peso vivo y peso canal caliente}

En la Figura 1 se muestran los valores individuales de peso vivo al sacrificio y peso de la canal caliente de cada lote de cabritos estu- 


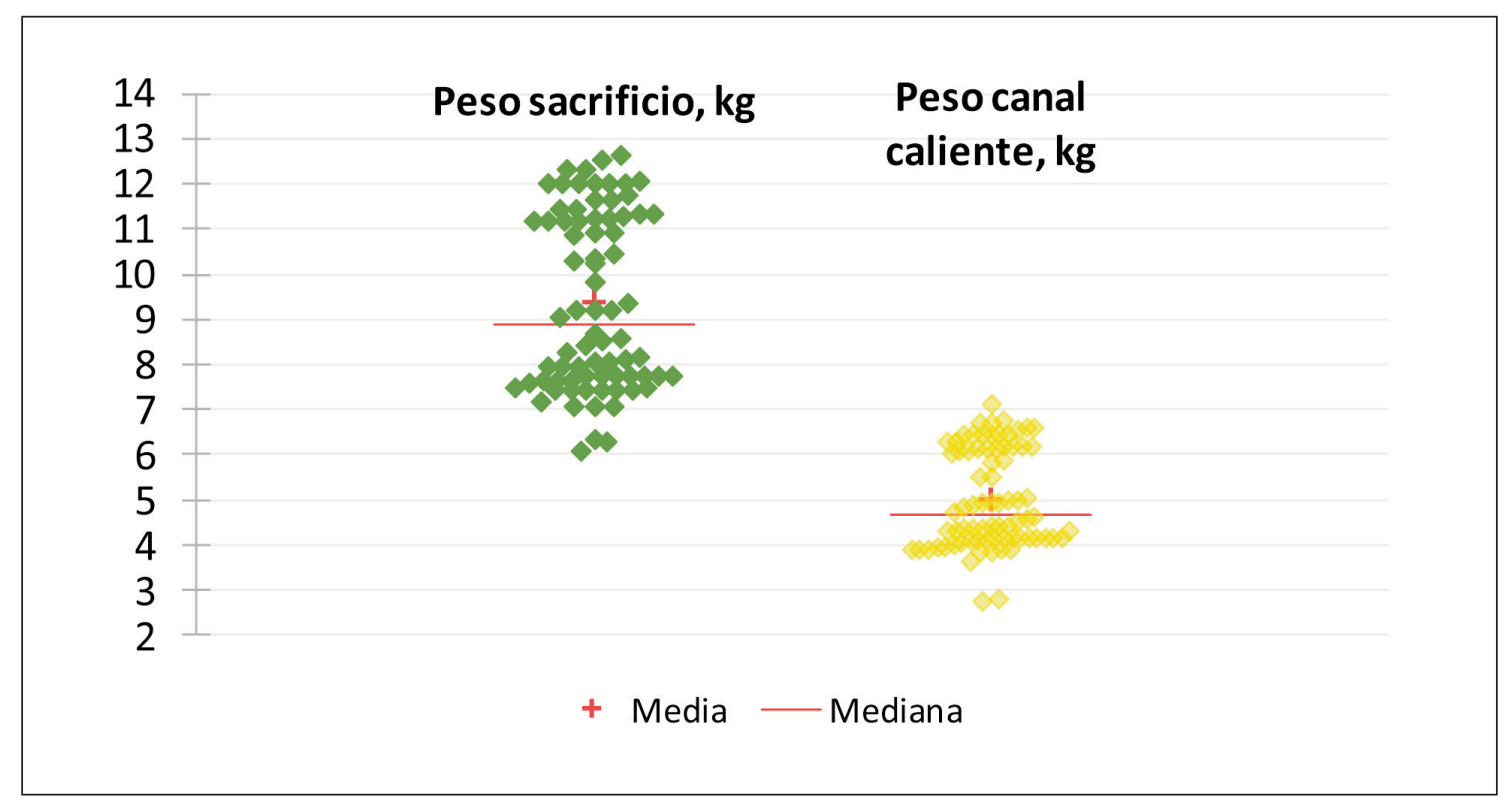

Figura 1. Peso vivo al sacrificio y peso canal caliente de la bibliografía.

Figure 1. Slaughter and hot carcass weights.

diados en la bibliografía. El intervalo de peso vivo va desde $6 \mathrm{~kg}$ hasta $13 \mathrm{~kg}$, y se puede observar en la distribución cómo hay dos grupos de peso divididos aproximadamente por la media, alrededor de los $9 \mathrm{~kg}$ de peso vivo. Respecto del peso de la canal caliente, el intervalo va desde los $3,5 \mathrm{~kg}$ hasta los $7 \mathrm{~kg}$, aunque hay dos lotes con pesos inferiores a $3 \mathrm{~kg}$ (Castro et al., 2008). Al igual que se observa en el peso vivo, hay dos grupos de canales diferenciadas: las canales con pesos superiores a $5 \mathrm{~kg}$ y las ligeras, con pesos inferiores a $5 \mathrm{~kg}$. Estos dos tipos de canal se corresponden con la legislación española que regula el etiquetado facultativo de la canal caprina y que establece el límite entre las categorías de "Lechal" y "Lechal ligero" en 5 kg (BOE, 2011). Respecto al peso canal de los artículos de esta revisión, el estudio griego utilizó cabritos con un peso canal mayor de $5 \mathrm{~kg}$, mientras que las canales del estudio turco pesaron menos de $5 \mathrm{~kg}$. En los estudios españoles, el
$55 \%$ de los lotes incluyó cabritos de menos de $5 \mathrm{~kg}$, mientras que los lotes de estudios italianos tuvieron animales con pesos menores de $5 \mathrm{~kg}$ en un $40 \%$.

\section{Composición proximal}

En la Tabla 1 se muestra la composición proximal de la carne de cabrito de la bibliografía consultada. Se observa cómo no hay grandes diferencias ni en humedad ni en proteína ni en cenizas. Sin embargo, el porcentaje de grasa varía mucho en función del peso canal caliente, aunque el porcentaje es bajo comparado con otras especies; al ser la especie caprina una especie poco precoz (Norman y Lawrie, 1991) deposita en mayor medida la grasa entorno a las vísceras (Chilliard et al., 1981; Dhanda et al., 2003), antes que en los depósitos subcutáneo o intramuscular. Respecto al sistema de lactancia utilizado en la bibliografía, no se observó ninguna varia- 
Tabla 1. Composición proximal de la carne de cabrito lechal en función del peso de la canal caliente.

Table 1. Proximal composition of suckling kid meat at two hot carcass weights.

\begin{tabular}{lcc}
\hline & \multicolumn{2}{c}{ Peso de la canal caliente } \\
\cline { 2 - 3 } & $<5 \mathrm{~kg}$ & $\geq 5 \mathrm{~kg}$ \\
\hline Humedad, \% & $76,66[76,12-77,20]$ & $75,70[74,79-76,61]$ \\
Proteína, \% & $20,79[19,89-21,69]$ & $19,72[18,86-20,58]$ \\
Grasa, \% & $1,60[1,16-2,05]$ & $2,64[1,59-3,69]$ \\
Cenizas, \% & $1,04[0,95-1,13]$ & $1,14[1,05-1,22]$ \\
\hline
\end{tabular}

Media e intervalo de confianza al $95 \%$ [entre corchetes]. Porcentajes expresados sobre materia fresca.

ción entre los valores de composición proximal de la carne de cabritos alimentados con lactoreemplazantes ( $17 \%$ de los lotes estudiados) o leche natural ( $83 \%$ de los lotes estudiados).

\section{pH y glucolisis}

El pH último en la mayoría de las especies de abasto está en el rango de 5,5 a 5,7 (Hopkins y Fogarty, 1998; Webb et al., 2005; Scheffler et al., 2011). Como se muestra en la Figura 2 tres de los lotes de cabrito lechal encontrados en la bibliografía tienen un valor de $\mathrm{pH}$ de 5,5 o menor, mientras que el $65 \%$ de los lotes tuvieron valores iguales o superiores a 5,7 . En esta misma figura se observa cómo los valores de $\mathrm{pH}$ son similares independientemente de la alimentación del cabrito, ya que los cabritos alimentados con lactoreemplazantes presentaron una mediana de 5,6 y los alimentados con leche materna presentaron una mediana de 5,8. De acuerdo con los estándares de algunos países, un $\mathrm{pH}>5,7$ se puede tener repercusiones en la calidad de la carne bovina (Thompson, 2002), y un pH > 6 produce carne oscura en rumiantes (Van Laack et al., 2001). Sin embargo, otros autores como
Ponnampalam et al. (2017) consideran normales los $\mathrm{pH}$ menores de 5,8 y elevados cuando son mayores de 6,2. Una hipótesis para explicar la alta frecuencia de aparición de $\mathrm{pH}$ mayor de 5,7 en el cabrito lechal podría ser la velocidad de la glucólisis. Como las canales de cabrito son muy pequeñas, en las condiciones de refrigeración habituales en los mataderos de ganado de abasto, estas se enfriarían muy rápidamente y por ello, sufrirían de una glucólisis post-mortem muy lenta (Pophiwa et al., 2020). Sin embargo, no se apreció una relación del pH último con el peso de la canal, por lo menos en este rango de pesos (Figura 2, derecha). La explicación más probable sea que los cabritos llegan al sacrifico con poco glucógeno muscular ya que la especie caprina es muy excitable (Casey y Webb, 2010) y sensible al estrés del manejo antes del sacrificio (Ripoll et al., 2011).

\section{Color y capacidad de retención de agua}

En la superficie de la carne fresca en contacto con el aire, la forma química predominante de la mioglobina es la oximioglobina, La oximioglobina tiene el color rojo brillante que aprecia el consumidor porque lo relaciona con frescura (Mancini y Hunt, 2005). 


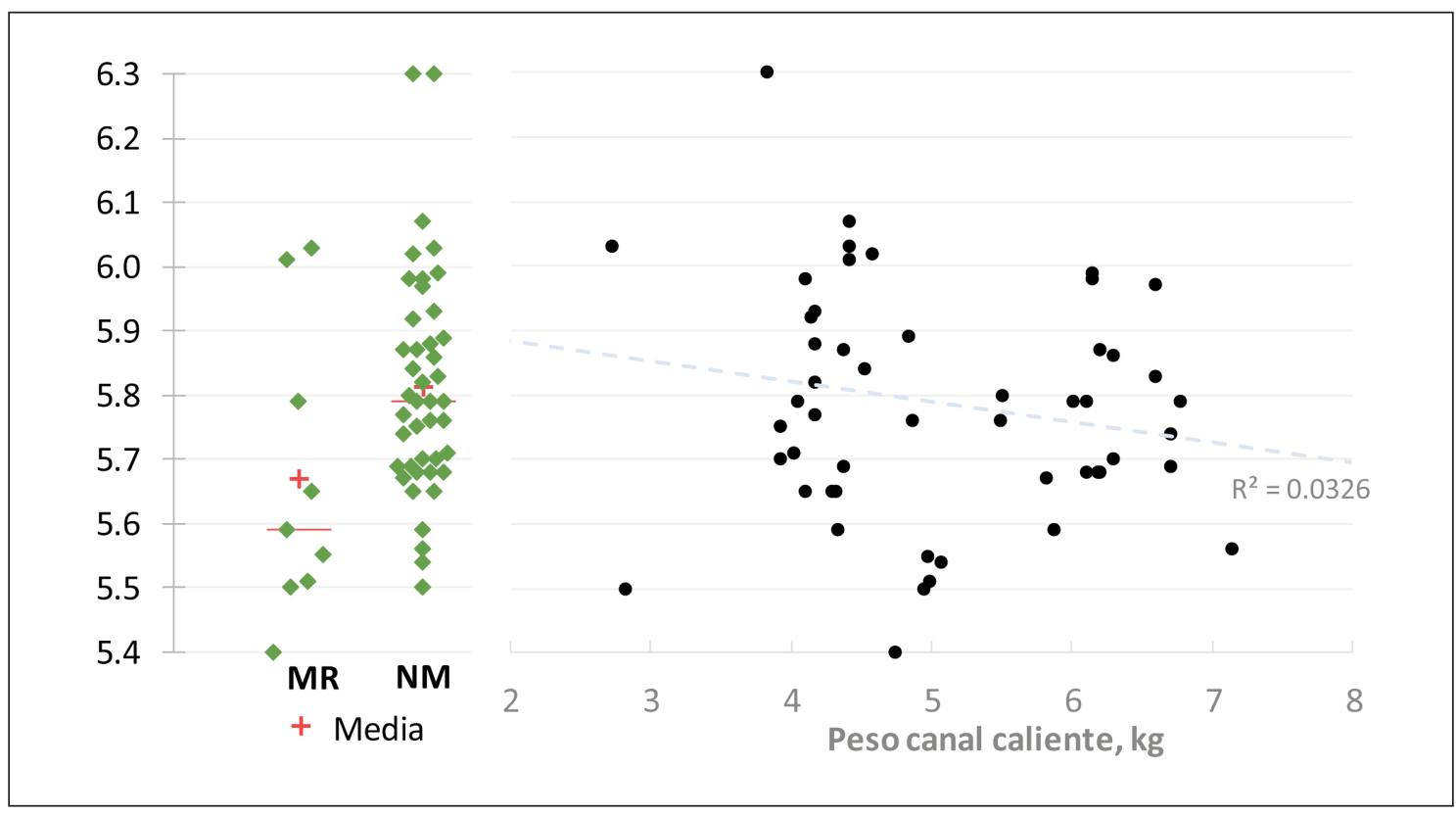

Figura 2. Valores de pH en función del sistema de lactancia (izquierda) y del peso de la canal (derecha) de la bibliografía consultada.

MR, lactoreemplazantes; NM, leche de la madre.

La línea azul discontinua es la recta de regresión entre el peso canal caliente y el pH.

Figure 2. $\mathrm{pH}$ values by rearing system (left) and carcass weight (right).

$M R$, milk replacers; NM, natural milk.

Dashed blue line is the regression line relating carcass weight and $\mathrm{pH}$.

La carne de cabrito lechal se caracteriza por tener una baja cantidad de pigmentos hemínicos en comparación con la de otras especies. Así, la carne de cabrito lechal puede tener valores de menores de 1,3 mg/g (Bañón et al., 2006), mientras que el ganado caprino mayor tienen valores superiores a 2,3 mg/g (Kesava Rao et al., 2003). La cantidad de pigmentos en la carne también es importante porque modifica la relación entre las variables instrumentales de color. Existe una relación positiva y significativa pero no lineal entre la cantidad de pigmentos hemínicos (más allá de su estado oxidativo) con la luminosidad y el tono, de forma que el mismo incremento en el tono produce un incremento pequeño o grande en la luminosidad si la carne es rica o pobre en pigmentos hemínicos, respectivamente (MacDougall, 1982). Este fenómeno se da también en el tono puesto que existe una relación positiva en la carne de cabrito lechal entre la luminosidad y el tono (Ripoll et al., 2012). En la Figura 3 podemos comprobar esa relación positiva entre la luminosidad y el tono, ya que cuando aumenta el tono también lo hace la luminosidad. Sin embargo, también se puede observar cómo se definen dos tipos de carne de cabrito, en función de esa relación positiva. En un grupo de carnes de cabrito, la luminosidad aumentó muy rápidamente con el incremento de tono mientras que en el otro grupo la luminosidad aumentó en menor 

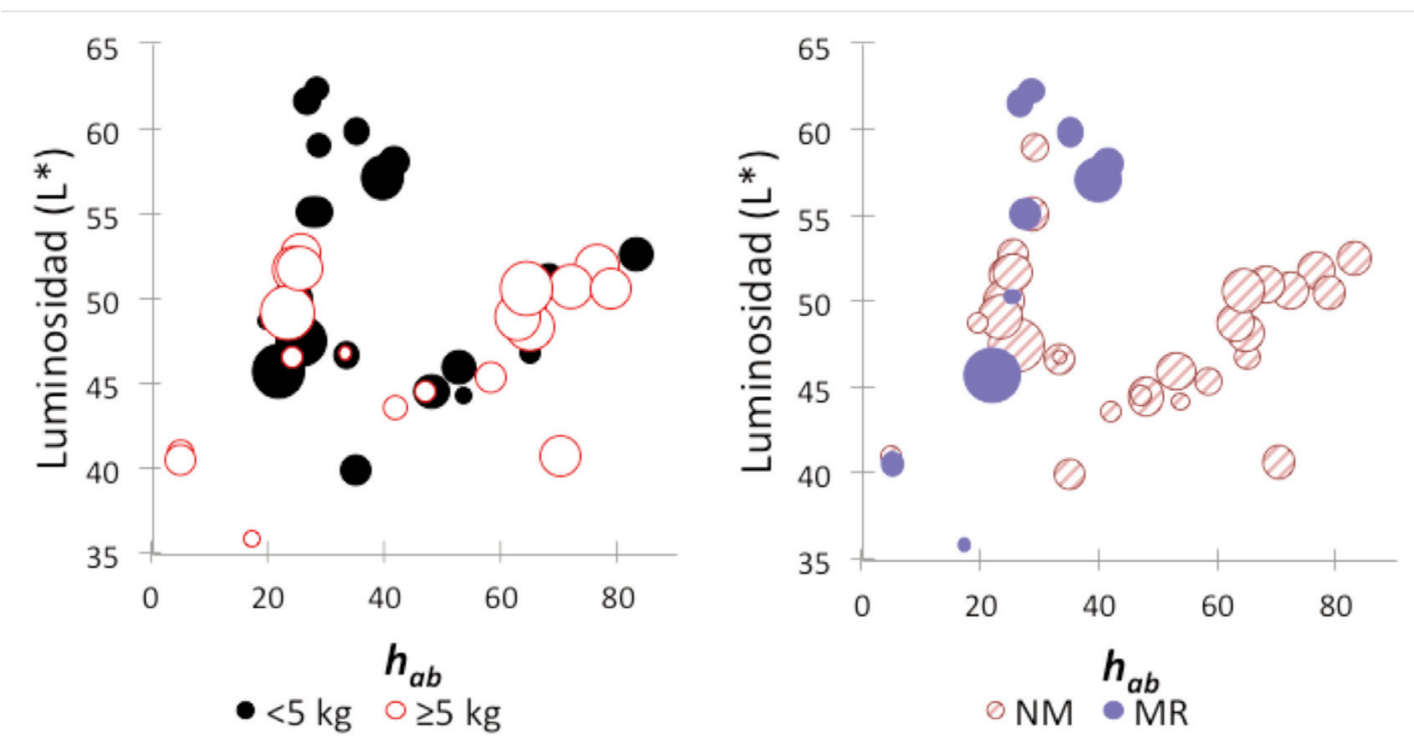

Figura 3. Luminosidad $\left(L^{*}\right)$, tono $\left(h_{a b}\right)$ y saturación $\left(C_{a b}^{*}\right)$ de la carne de cabrito lechal según su peso de canal caliente y el sistema de lactancia.

MR, lactoreemplazantes; NM, leche de la madre. La saturación está representada por el tamaño de los puntos.

Figure 3. Lightness $\left(L^{*}\right)$, hue angle $\left(h_{a b}\right)$ and chroma $\left(C_{a b}^{*}\right)$ of suckling kid meat by carcass weight and rearing system.

MR, milk replacers; NM, natural milk. The size of dots shows the value of chroma.

medida con el incremento del tono. En la Figura 3 izquierda se observa como estos dos grupos de carne no están definidos por el peso de la canal caliente, ya que en los dos grupos encontramos canales más ligeras y más pesadas de $5 \mathrm{~kg}$. Sin embargo, en la Figura 3 derecha sí se observa cómo el grupo con menor pendiente, en el que la luminosidad aumenta menos con el aumento del tono, está formado exclusivamente por animales alimentados con leche natural. Sin embargo, en el grupo de pendiente mayor, donde la luminosidad aumenta más con el aumento del tono, se observan animales de lactancia artificial y natural mezclados. Parece pues que los lactoreemplazantes dan lugar a una carne que se comporta de manera particular, con grandes cambios de luminosidad cuando cambia el tono, aunque esto no está claro y habría que indagar más en futuras investigaciones.

Por otra parte, se podría pensar que estos dos grupos podrían deberse a diferencias metodológicas en la medida del color. Sin embargo, todos los estudios utilizan aparatos comparables del mismo fabricante, excepto CaputiJambrenghi et al. (2010). La mayoría de los artículos utilizan el espacio de color CIELab, mientras que dos de ellos utilizaron el espacio HunterLab. Yalcintan et al. (2018a) encontraron que la carne de cabritos alimentados con leche natural tuvo mayor luminosidad y tono que los de lactancia artificial. En el estudio de Bañón et al. (2006), la carne de lactancia artificial tuvo menos saturación y ma- 
yor tono que la de lactancia natural. Sin embargo, Moreno-Indias et al. (2012) encontraron que el color no se modificaba por el lactoreemplazante. Por otra parte, De Palo et al. (2015) encontraron que las diferencias en color dependían del tipo de lactoreemplazante. A nivel de estudios individuales parece que no hay una conclusión clara al respecto de la influencia del tipo de lactancia ya que puede estar condicionada por las razas usadas y el tipo de lactoreemplazante. Sin embargo, al agrupar los datos de todos los estudios, se aprecia una influencia del sistema de lactancia, que actúa de distinta forma en función de los valores de luminosidad y tono de la carne.

En la Figura 4 se representan los valores encontrados en la bibliografía para las coordenadas $a *, b *$ y $L^{*}$, representada esta última por el tamaño del punto. El peso de la canal caliente (Figura 4, izquierda) no influyó la distribución de estos parámetros de color, estando la carne de los dos pesos de canal repartidos homogéneamente en función de a* y $b$ *. Sin embargo, en los datos segregados por sistemas de lactancia (Figura 4, derecha) se observa una clara tendencia a que la carne con valores más altos de $b$ * (por encima de 8) provenga de animales alimentados con lactancia natural, mientras que por debajo de este valor encontramos carnes de cabritos alimentados en los dos sistemas de lactancia. De forma similar, pero menos clara, vemos que la carne con valores de a* menores de 6 es de animales de lactancia natural mientras que por encima de ese valor corresponde a animales criados en cualquiera de los dos sistemas. De acuerdo con esto, algunos autores encontraron que la carne de cabritos y corderos lechales alimentados con leche materna tuvo mayor tono, es decir, mayor índice

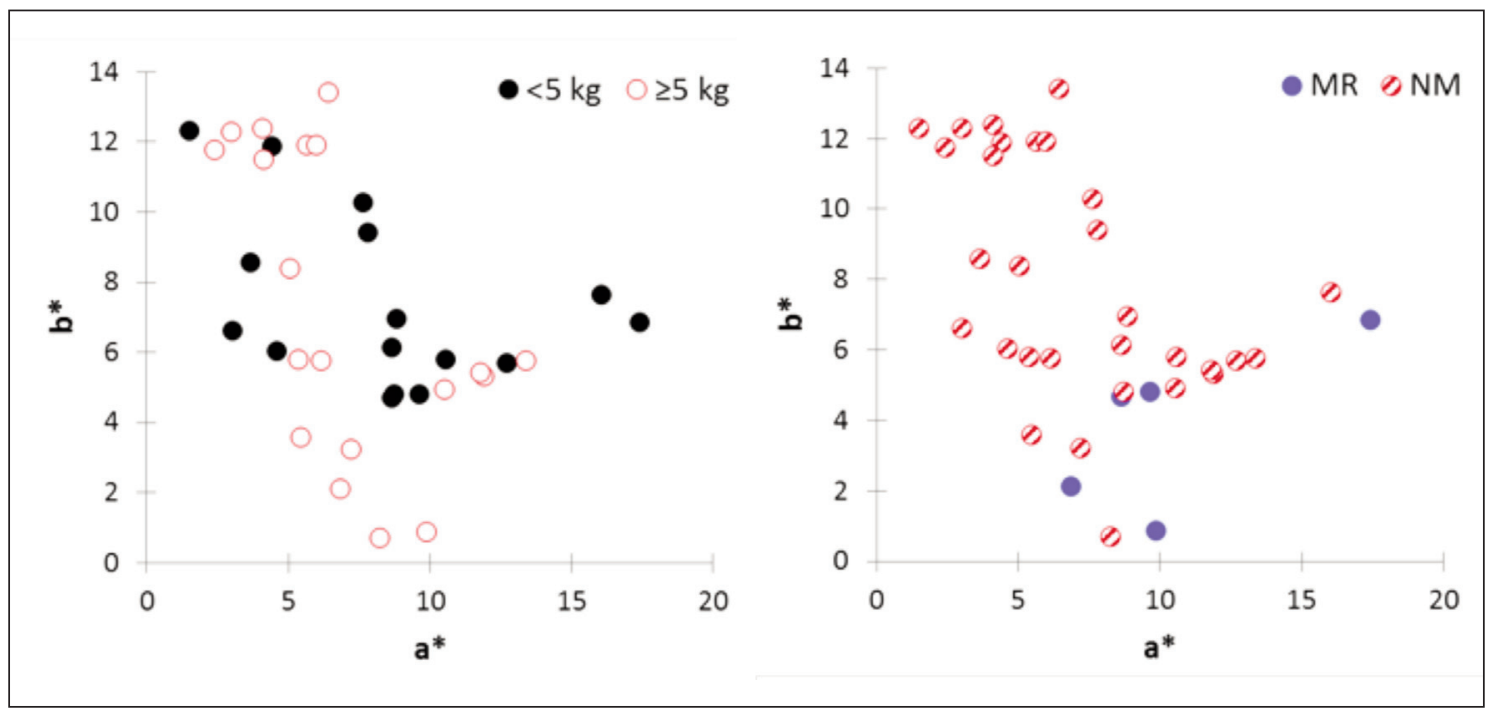

Figura 4. Índices de rojo $\left(a^{*}\right)$ y amarillo $\left(b^{*}\right)$ de la carne de cabrito lechal según su peso de canal caliente (izquierda) y el sistema de lactancia (derecha).

MR, lactoreemplazantes; NM, leche de la madre.

Figure 4. Red ( $\left.a^{*}\right)$ and yellow ( $\left.b^{*}\right)$ indexes of suckling kid meat by carcass weight (left) and rearing system (right).

$M R$, milk replacers; NM, natural milk. 
de $b$ * menor de a*, que la carne de animales alimentados con lactoreemplazantes (Osorio et al., 2008; De Palo et al., 2015; Yalcintan et al., 2018b). Estos autores justifican estas diferencias a diferencias en la cantidad de grasa intramuscular. Otros autores señalan que la leche materna puede tener deficiencias de hierro (Sañudo et al., 2012).

\section{Textura instrumental}

Existe poca bibliografía que recoja datos de textura de carne de cabrito lechal en general, y aún menos de animales alimentados con lactoreemplazantes. En la Figura 5 se muestran los resultados de la fuerza de cizalla Warner-Bratzler y del método de compresión encontrados en la bibliografía. Mientras que múltiples estudios usaron el método de Warner-Bratzler, solo 2 estudios usaron el test de compresión (Ripoll et al., 2012; Guerrero et al., 2016). En la Figura 5 vemos cómo los valores de fuerza Warner-Bratzler oscilan entre los $9 \mathrm{~N}$ y los $70 \mathrm{~N}$ aproximadamente, aunque la mayoría de los datos se encuentran en la horquilla de $25 \mathrm{~N}$ a $55 \mathrm{~N}$, con una media de $37,5 \mathrm{~N}$. Los valores mayores de $55 \mathrm{~N}$ corresponden a los estudios de un mismo equipo (Guzmán et al., 2019a y 2019b). Sin embargo, estos valores más altos no se pueden achacar a diferencias en la metodología. Este equipo usó una metodología estándar con un tiempo de cocinado de $30 \mathrm{~min}$ a $75^{\circ} \mathrm{C}$. Las temperaturas de cocinado de otros estudios oscilan entre $70{ }^{\circ} \mathrm{C}$ y $85^{\circ} \mathrm{C}$ durante un tiempo de $30 \mathrm{~min}$ a $45 \mathrm{~min}$. Dos artículos cocinan la carne hasta una temperatura en el centro de la muestra de $75^{\circ} \mathrm{C}$ y uno hasta $70^{\circ} \mathrm{C}$. Respecto a la forma de hacer las submuestras, se utilizan los dos sistemas más extendidos, con

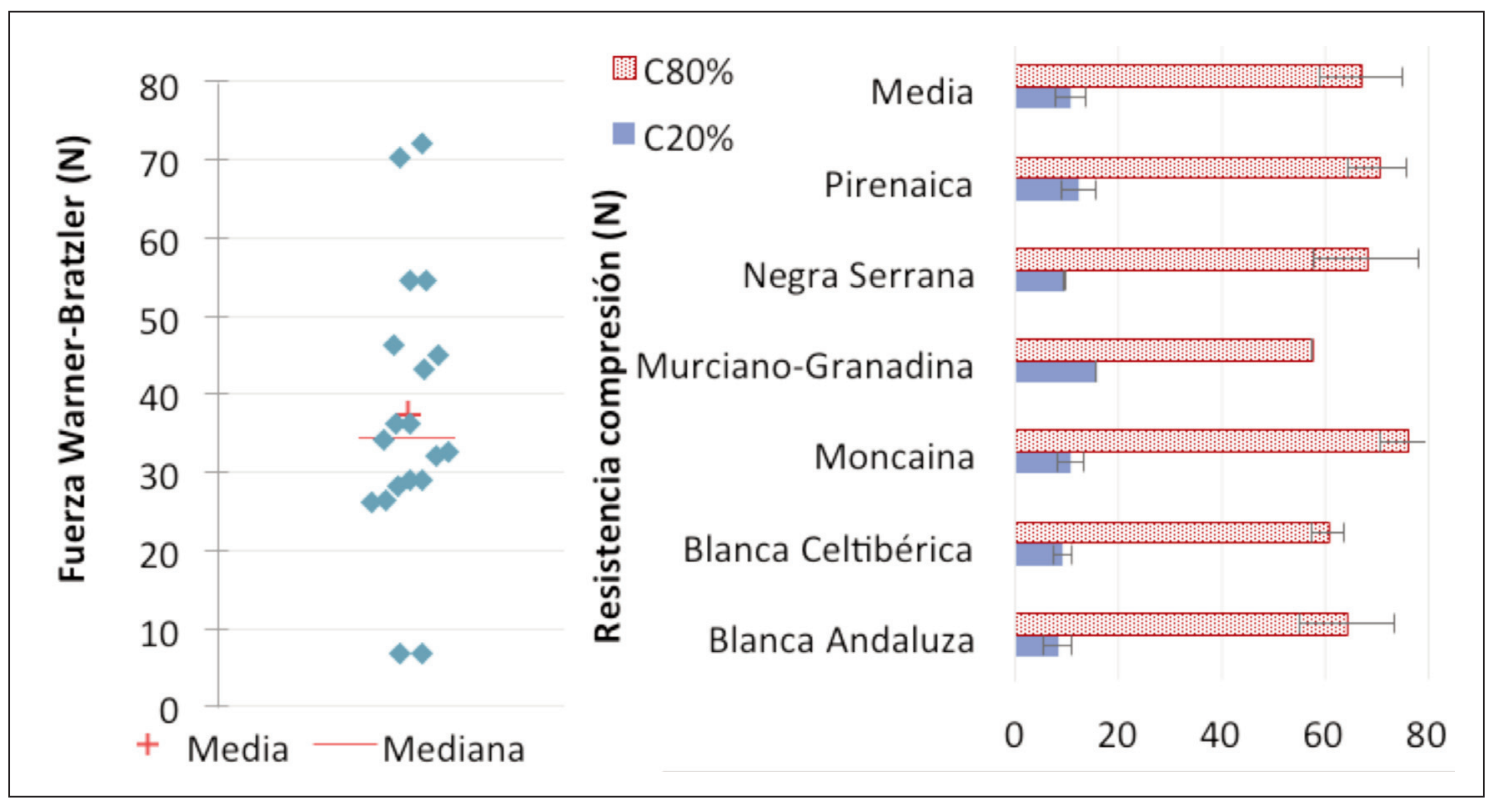

Figura 5. Fuerza máxima de cizalla Warner-Bratzler en Newtons (izquierda) y resistencia a la compresión en Newtons (derecha) de carne de cabrito lechal.

Figure 5. Warner-Bratzler shear force in Newtons (left) and compression force in Newtons (right) of suckling kid meat. 
submuestras de sección circular o cuadrada, pero esto no influyó en la distribución de los valores de la dureza. Miller et al. (2001) trabajando con vacuno, encontraron que para los consumidores, la transición entre carne dura y tierna ocurre entre los $42 \mathrm{~N}$ y $48 \mathrm{~N}$. Otros autores (Webb et al., 2005) encontraron valores similares a los de Miller et al. (2001). Así pues, vemos cómo la mayor parte de la carne de cabrito lechal se puede considerar tierna. La principal causa de la dureza es la cantidad de enlaces cruzados resistentes al calor del epi-, peri- y endomisio. El colágeno de los animales viejos es menos soluble que el de los jóvenes así que cuando la carne se cocina se solubiliza menos, aunque se debiliten los enlaces cruzados, resultando en una carne más dura (Warris, 2000).

Respecto del método de compresión, se observa cómo hay diferencias entre razas tanto en la compresión al $20 \%$ como al $80 \%$. Sin embargo, esta comparación se limita a los datos de dos estudios. Este método proporciona una información muy útil sobre la textura de la carne, pero su uso no está tan extendido como el de Warner-Bratzler. Está ampliamente aceptado que la compresión a ratios bajas, como el $20 \%$, mide la resistencia mecánica debida a la miofibra muscular ya que con esta ratio de compresión el tejido conectivo no se comprime, sino que solo se pliega, mientras que la compresión a ratios altas $(80 \%)$ sí que mide la aportación a la dureza del tejido conectivo (Lepetit, 1989). El pH también influye sobre la dureza y lo hace tanto por el valor del pH último como por la velocidad a la que se desarrolla la glucólisis post-mortem. Así, la dureza es mínima cuando el valor de $\mathrm{pH}$ último se alcanza a una velocidad moderada, mientras que si es muy rápida o muy lenta la dureza es mayor (Smulders et al., 1990). Entre los factores estudiados en los artículos de la revisión se encuentran el peso del animal, que no modificó la dureza medida con la célula Warner-Bratzler según
Juárez et al. (2009) y Yalcintan et al. (2018a), aunque Ripoll et al. (2012) sí que encontraron que la carne de canales más pesadas fue menos dura. El uso de lactoreemplazantes tampoco afectó a la dureza (Moreno-Indias et al., 2012; De Palo et al., 2015; Yalcintan et al., 2018a), al igual que la cría en granjas ecológicas o el sexo (Guzmán et al., 2019a y 2019b). Sin embargo, Caputi-Jambrenghi et al. (2010) encontraron que la intensificación del sistema de manejo de las madres si modificó la textura de la carne del cabrito. Otros efectos importantes fueron el manejo estresante de los animales y la duración del transporte al matadero (Alcalde et al., 2017).

\section{Ácidos grasos y compuestos aromáticos}

En las Figuras 6 y 7 se muestran los porcentajes del total de ácidos grasos saturados e insaturados y de la ratio n-6/n-3. En la Figura 6 se observa cómo el porcentaje de ácidos grasos saturados va desde $38,1 \%$ hasta un $54,8 \%$, mientras que los insaturados estuvieron entre el $45,2 \%$ y el $60,6 \%$. Por estar muy desequilibrado el número de estudios no se pueden comparar sistemas de lactancia, pero se observa en la Figura 6 cómo, en general, el porcentaje de ácidos grasos saturados de la carne de canales muy ligeras $(<5 \mathrm{~kg})$ se mueve en el intervalo $41,2 \%$ a $54,8 \%$ mientras que la carne de canales ligeras ( $\geq 5 \mathrm{~kg}$ ) presentaron valores inferiores, en el intervalo de $38,1 \%$ a $46,2 \%$. Evidentemente, la lectura de los ácidos grasos insaturados fue la inversa ya que los datos son complementarios. Los estudios que compararon el uso de lactoreemplazantes con leche materna concluyeron que los lactoreemplazantes aumentaron el porcentaje de ácidos grasos insaturados disminuyendo el de saturados (De Palo et al., 2015; Tsiplakou et al., 2016). La correlación entre el porcentaje de grasa intramuscular y el porcentaje de ácidos grasos saturados no fue significativa $(p>0,05)$. Esta ausencia de correlación pudo ser debida 


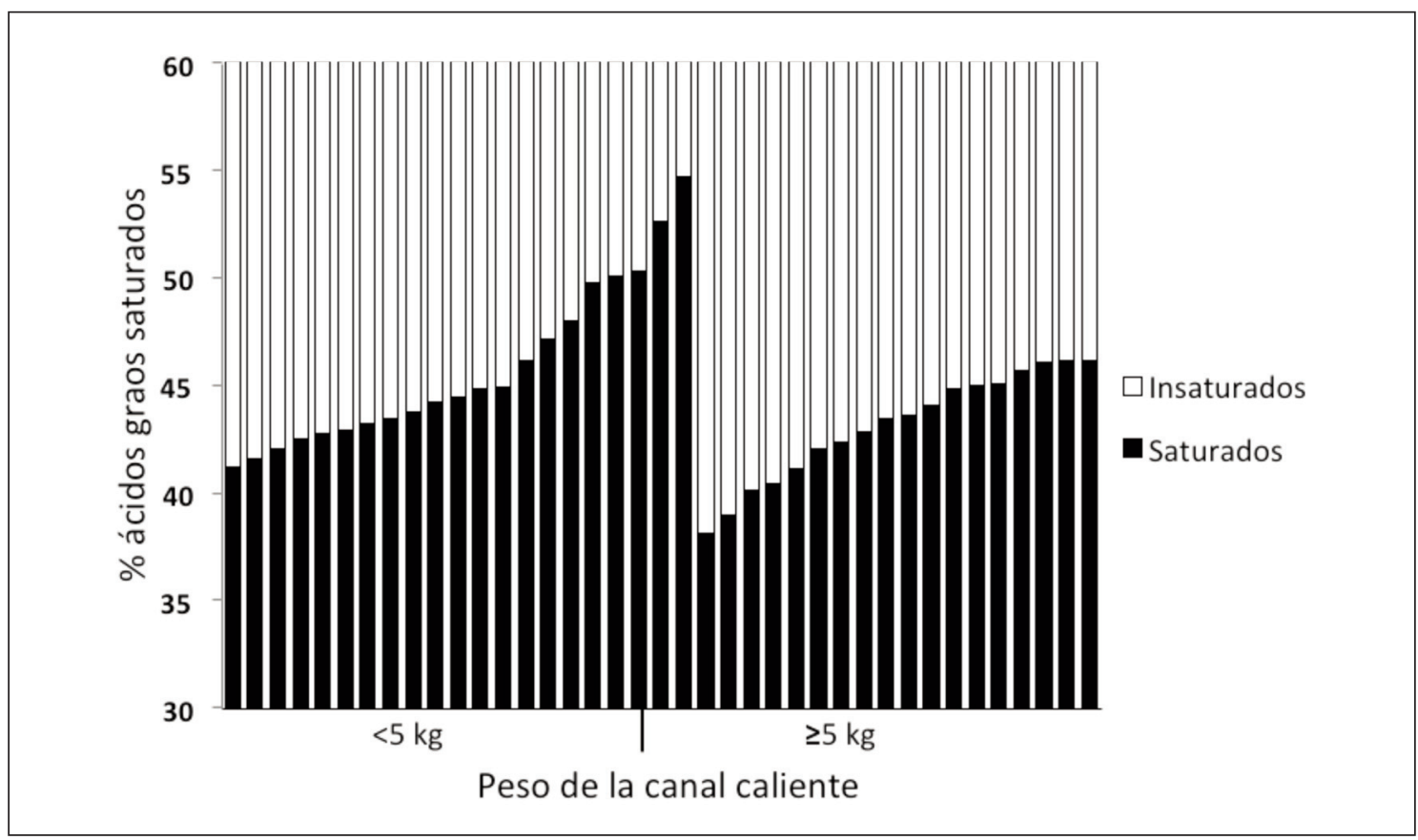

Figura 6. Porcentaje de ácidos grasos saturados e insaturados de la grasa intramuscular en función del peso de canal caliente.

Figure 6. Percentage of saturated and unsaturated fatty acids of intramuscular fat by hot carcass weight.

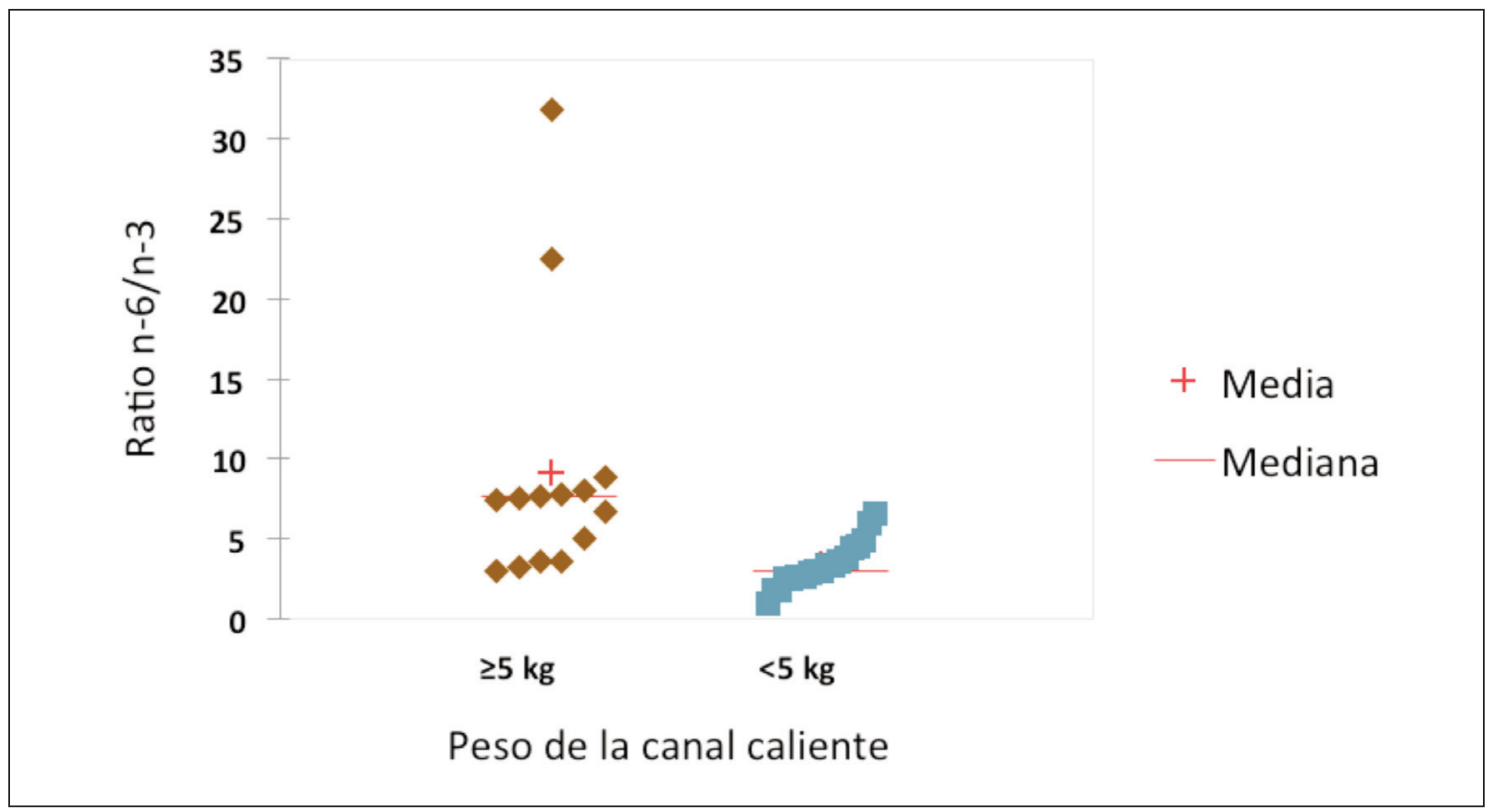

Figura 7. Ratio $n-6 / n-3$ de los ácidos grasos de la grasa intramuscular.

Figure 7. n-6/n-3 ratio of fatty acids of intramuscular fat. 
principalmente a la escasa cantidad de grasa intramuscular y el pequeño rango de valores, aunque otros factores como cuestiones metodológicas y el sistema de manejo y alimentación de los animales también pudieron afectar.

Respecto de la relación de los ácidos grasos insaturados $n-6 / n-3$, se observa en la bibliografía que la ratio $n-6 / n-3$ de la grasa intramuscular de canales de cabrito lechal con peso menor a $5 \mathrm{~kg}$ fue menor $(3,4)$ que la de la grasa de canales con peso mayor a $5 \mathrm{~kg}$ $(9,0)$. Sin embargo, como se observa en la Figura 7, esta media está influida por los valores de los dos lotes de cabritos presentados por Tsiplakou et al. (2016), que fueron mucho mayores que el resto. Sin tener en cuenta este estudio, el rango de valores para la ratio $\mathrm{n}-6 / \mathrm{n}-3$ de las canales de menos de $5 \mathrm{~kg}$ fue de 1,0 a 6,6 mientras que para las canales de más de $5 \mathrm{~kg}$ fue de 3,1 a 8,9; así que las canales más ligeras siguen teniendo una ratio más favorable desde el punto de vista saludable, aunque en general tenían mayor porcentaje de ácidos grasos saturados. Los estudios que compararon el uso de lactoreemplazantes con leche materna concluyeron que los lactoreemplazantes aumentaron relación n-6/n-3 (De Palo et al., 2015; Tsiplakou et al., 2016). Las recomendaciones nutricionales comúnmente aceptadas para los humanos son la disminución del contenido graso en la dieta, una mayor ingesta de ácidos grasos insaturados, evitar la configuración trans, y que esta grasa tenga una relación de ácidos grasos poliinsaturados $n-6 / n-3$ inferior a 4 (Wood et al., 2004).

En la bibliografía estudiada no se han encontrado resultados de ácidos grasos de cadena ramificada. A la vista de los resultados de los estudios de la bibliografía recopilada, no queda claro si este tipo de ácidos grasos ramificados no se han encontrado porque no se hallan presentes en la carne de cabrito o por la metodología empleada, p.ej. la longitud de la columna cromatográfica emple- ada. Otro problema metodológico a la hora de comparar estudios es que, al estar los resultados de los ácidos grasos individuales en porcentaje respecto del total de ácidos grasos detectados, estos resultados son dependientes de la precisión y el número de ácidos grasos detectados.

\section{Conclusiones}

Esta revisión resume los principales resultados encontrados en la bibliografía sobre la calidad instrumental del músculo longissimus thoracis et lumborum de cabritos de menos de $13 \mathrm{~kg}$ de peso vivo, alimentados exclusivamente con leche, ya sea natural o artificial. Sin embargo, debido a la escasa información encontrada las conclusiones obtenidas deben servir para orientar futuros estudios.

Las canales se distribuyeron en dos grandes grupos por peso separadas por el umbral de $5 \mathrm{~kg}$. Estos dos grupos concuerdan con los pesos de canal que con más frecuencia se comercializan en España, canales más pesadas en el Norte y más ligeras en el Sur. La carne de canales más pesadas tuvo mayor contenido en grasa intramuscular con mayor porcentaje de ácidos grasos insaturados. Sin embargo, no se apreció una influencia del peso de la canal sobre el $\mathrm{pH}$, el porcentaje de proteína, la dureza ni el color instrumental.

El pH de la carne de cabrito lechal tiende a ser alto, con gran parte de los datos mayores de 5,7 , pero no se ha encontrado efecto del $\mathrm{pH}$ sobre los parámetros de calidad.

El sistema de lactancia no modificó la composición proximal de la carne, ni su pH o textura pero si influyó en el color de la carne. La carne de cabritos alimentados con leche natural presentó un índice de amarillo más alto que la carne de cabritos alimentados con lactoreemplazantes. También se observó que el 
uso de lactoreemplazantes aumentó el porcentaje de ácidos grasos insaturados y la relación n-6/n-3.

Existe muy poca información científica sobre la calidad instrumental de la carne de cabritos ligeros y la mayoría de la bibliografía es española. Teniendo en cuenta que los problemas estructurales del sector sitúan a 19 de las 22 razas autóctonas españolas al borde de la extinción, serían necesarios más estudios para cubrir esta laguna de conocimiento.

\section{Agradecimientos}

Los autores agradecen a los ganaderos, las asociaciones de ganaderos y al personal del CITA de Aragón por su colaboración. Este estudio ha sido financiado por el Ministerio de Economía y Competitividad del Gobierno de España y FEDER (RTA2012-23-C3), y los fondos para los grupos de investigación del Gobierno de Aragón (A14-17R SAGAS).

\section{Material complementario}

El material complementario de este artículo se puede consultar en la URL https://doi.org/ 10.12706/itea.2020.016

\section{Referencias bibliográficas}

Alcalde MJ, Ripoll G, Panea B (2010). La producción de carne caprina en Andalucía. En: La producción de carne en Andalucía. (Eds. Horcada AL), pp. 317-340. Junta de Andalucía. Sevilla, España.

Alcalde MJ, Suárez MD, Rodero E, Álvarez R, Sáez MI, Martínez TF (2017). Effects of farm management practices and transport duration on stress response and meat quality traits of suckling goat kids. Animal 11: 1626-1635. https:// doi.org/10.1017/S1751731116002858
Bañón S, Vila R, Price A, Ferrandini E, Garrido MD (2006). Effects of goat milk or milk replacer diet on meat quality and fat composition of suckling goat kids. Meat Science 72: 216-221. https://doi. org/10.1016/j.meatsci.2005.07.004

BOE (2011). Resolución de 19 de diciembre de 2011, de la Dirección General de Recursos Agrícolas y Ganaderos, por la que se aprueba la guía del etiquetado facultativo de carne de cordero y cabrito. Boletín Oficial del Estado núm. 314, de 30 de diciembre de 2011, pp. 146362-146367.

Caputi-Jambrenghi A, Colonna M, Giannico F, Coluccia A (2010). Meat quality in suckling kids reared by differentproduction systems. Progress in Nutrition 11: 36-46.

Casey NH, Webb EC (2010). Managing goat production for meat quality. Small Ruminant Research 89: 218-224. https://doi.org/10.1016/j. smallrumres.2009.12.047

Castel JM, Mena Y, Ruiz FA, Gutiérrez R (2012). Situación y evolución de los sistemas de producción caprina en España. Tierras Caprino 1: 24-37.

Castro N, Sanchez-Macias D, Moreno-Indias I, Morales-DelaNuez A, Arguello A, Capote J (2008). Effects of milk replacer supplementation with conjugated linoleic acid and live weight at slaughter on growth and carcass and meat quality of kids. Journal of Animal and Veterinary Advances 7: 196-202.

Chamorro A, Miranda FJ, Rubio S, Valero V (2012). Innovations and trends in meat consumption: An application of the delphi method in Spain. Meat Science 92: 816-822. https://doi.org/10. 1016/j.meatsci.2012.07.007

Chilliard Y, Sauvant D, Bas P, Pascal G, MorandFehr $P$ (1981). Importance relative et activités métaboliques des différents tissus adipeux de la chèvre laitière. En: Nutrition and systems of goat feeding (Eds. Morand-Fehr P, Bourbouze A y Simiane M), pp. 90-100. ITOVIC-INRA, Paris, Francia.

De Palo P, Maggiolino A, Centoducati N, Tateo A (2015). Effects of different milk replacers on carcass traits, meat quality, meat color and fatty acids profile of dairy goat kids. Small Ruminant Research 131: 6-11. https://doi.org/10. 1016/j.smallrumres.2015.09.001 
Dhanda J, Taylor D, Murray P, Pegg R, Shand P (2003). Goat meat production: Present status and future possibilities. Asian-Australasian Journal of Animal Science 16: 1842-1852. https:// doi.org/10.5713/ajas.2003.1842

Eurostat. (2019). Eurostat. Agricultural statistics. Statistical Office of the European Communities. Disponible en: appsso.eurostat.ec.europa. eu/nui/setupDownloads.do (Consultado: 10 octubre 2019).

FAO (2019). Faostat, food and agriculture data. Disponible en: www.fao.org/faostat/en/ (Consultado: 10 octubre 2019).

Forrest J, Aberle D, Hedrick B, Judge D, Merkel A (1979). Fundamentos de ciencia de la carne. Editorial acribia. Zaragoza, España, 21 pp.

Guerrero A, Lemes JS, Campo MM, Olleta JL, Muela E, Resconi VC, Guerra VM, Assis-Macedo F, Sañudo C (2016). Características de la canal y de la carne en la raza caprina Bermeya. Comparación con el Ternasco de Aragón y lechales de la raza Murciano-Granadina. ITEA-Informacion Tecnica Economica Agraria 112: 271-285. https://doi.org/10.12706/itea.2016.017

Guzmán JL, De-La-Vega F, Angel Zarazaga L, Argüello A, Delgado-Pertíñez M (2019a). Carcase and meat quality of Blanca Andaluza kids fed exclusively with milk from their dams under organic and conventional grazing-based management systems. Italian Journal of Animal Science 18: 1186-1191. https://doi.org/10.1080/ 1828051X.2019.1638317

Guzmán JL, de la Vega F, Zarazaga LÁ, Argüello A, Delgado-Pertíñez M (2019b). Carcass characteristics and meat quality of conventionally and organically reared suckling dairy goat kids of the Payoya breed. Annals of Animal Science 19: 1143-1159. https://doi.org/10.2478/aoas2019-0047

Hopkins DL, Fogarty NM (1998). Diverse lamb genotypes-2. Meat $\mathrm{pH}$, colour and tenderness. Meat Science 49: 477-488. https://doi.org/ https://doi.org/10.1016/S0309-1740(98)00051-5

Juárez M, Micheo JM, García E, Peña F, Polvillo O (2009). Efecto del peso de la canal sobre la calidad de la carne de "Chivo Lechal Malagueño".
ITEA-Informacion Técnica Economica Agraria 105: 28-35.

Kesava Rao V, Kowale BN, Verma AK (2003). Effect of feeding water washed neem (Azadirachta indica) seed kernel cake on the quality, lipid profile and fatty acid composition of goat meat. Small Ruminant Research 47: 213-219. https://doi.org/10.1016/S0921-4488(02)00256-0

Lawrie RA (1998). Meat science $3^{\text {th }}$ ed. Acribia, S.A, Zaragoza, España, 367 pp.

Lepetit J (1989). Deformation of collagenous, elastin and muscle fibres in raw meat in relation to anisotropy and length ratio. Meat Science 26: 4766. https://doi.org/10.1016/0309-1740(89)90056-9

MacDougall DB (1982). Changes in the colour and opacity of meat. Food Chemistry 9: 75-88. https://doi.org/10.1016/0308-8146(82)90070-X

Mancini RA, Hunt MC (2005). Current research in meat color. Meat Science 71: 100-121. https:// doi.org/10.1016/j.meatsci.2005.03.003

MAPA (2019a).Datos estadísticos del ministerio de agricultura, pesca y alimentación. Disponible en: www.mapa.gob.es/es/estadistica/temas/ (Consultado: 14 octubre 2019).

MAPA (2019b).Encuesta de sacrificio de ganado del ministerio de agricultura y pesca, alimentación y medio ambiente. Resultados definitivos por provincias y comunidades autónomas de años anteriores (censo exhaustivo). Disponible en: www.mapama.gob.es/es/estadistica/temas/estadisticas-agrarias/ganaderia/encuestas-sacrificio-ganado/ (Consultado: 14 octubre 2019).

Marsico G, Vicenti A, Centoducati P, Braghieri A (1993). Influence of weaning age on productive performance of kids slaughtered at 107 days of age. Small Ruminant Research 12: 321-328. https://doi.org/10.1016/0921-4488(93)90067-R

Martín-Cerdeño VJ (2018). Consumo y gasto en carne y productos cárnicos. Distribución y Consumo 2: 5-14.

Miller MF, Carr MA, Ramsey CB, Crockett KL, Hoover LC (2001). Consumer thresholds for establishing the value of beef tenderness. Journal of Animal Science 79: 3062-3068. https://doi. org/10.2527/2001.79123062x 
Moreno-Indias I, Morales-de la Nuez A, Hernandez-Castellano L, Sanchez-Macias D, Capote J, Castro N, Argüello A (2012). Docosahexaenoic acid in the goat kid diet: Effects on immune system and meat quality. Journal of Animal Science 90: 3729-3738. https://doi.org/10.2527/ jas.2011-4351

Norman G, Lawrie R (1991). The potential of meat from the goat. En: Developments in meat science (Eds. Lawrie RA), pp. 57-87. Elsevier Applied Science, Londres, Reino Unido.

Osorio MT, Zumalacárregui JM, Cabeza EA, Figueira A, Mateo J (2008). Effect of rearing system on some meat quality traits and volatile compounds of suckling lamb meat. Small Ruminant Research 78: 1-12. https://doi.org/10.1016/j.smallrumres. 2008.03.015

Panea B, Ripoll G, Sañudo C, Alcalde MJ, Horcada A, Teixeira A (2008). Estudio integral del cabrito lechal español. Una propuesta de indicación geográfica protegida y de clasificación. Feagas 34: 61-66.

Panea B, Ripoll G, Sañudo C, Teixeira A, Horcada A, Alcalde MJ (2010). Estudio mediante encuesta del primer eslabón del canal de distribución de la carne de cabrito. XXXV Congreso de la Sociedad Española de Ovinotecnia y Caprinotecnia, 22-24 de septiembre de 2010. Valladolid, España, pp. 466-469.

Peña F, Herrera M, Subires J, Aparicio J (1985). Consumo de leche y crecimiento en peso vivo en chivos de raza Malagueña durante la fase de lactación. Archivos de Zootecnia 34: 301-314.

Ponnampalam EN, Hopkins DL, Bruce H, Li D, Baldi G, Bekhit AE-d (2017). Causes and contributing factors to "dark cutting" meat: Current trends and future directions: A review. Comprehensive Reviews in Food Science and Food Safety 16: 400-430. https://doi.org/10.1111/1541-4337.12258

Pophiwa P, Webb EC, Frylinck L (2020). A review of factors affecting goat meat quality and mitigating strategies. Small Ruminant Research 183: 106035. https://doi.org/10.1016/j.smallrumres.2019.106035

Price JF, Schweighet BS (1994). Ciencia de la carne y de los productos cárnicos. Editorial Acribia S.A, Zaragoza, España, 581 pp.
Ripoll G, Alcalde MJ, Horcada A, Panea B (2011). Suckling kid breed and slaughter weight discrimination using muscle colour and visible reflectance. Meat Science 87: 151-156. https://doi. org/10.1016/j.meatsci.2010.10.006

Ripoll G, Alcalde MJ, Horcada A, Campo MM, Sanudo C, Teixeira A, Panea B (2012). Effect of slaughter weight and breed on instrumental and sensory meat quality of suckling kids. Meat Science 92: 62-70. https://doi.org/10.1016/j.meatsci.2012.04.011

Sanz-Sampelayo M, Fernández J, Ramos E, Hermoso R, Extremera F, Boza J (2006). Effect of providing a polyunsaturated fatty acid-rich protected fat to lactating goats on growth and body composition of suckling goat kids. Animal Science 82: 337-344. https://doi.org/10.1079/ ASC200646

Sañudo C, Campo MM, Muela E, Olleta JL, Delfa R, Jiménez-Badillo R, Alcalde MJ, Horcada A, Oliveira I, Cilla I (2012). Carcass characteristics and instrumental meat quality of suckling kids and lambs. Spanish Journal of Agricultural Research 10: 690700. https://doi.org/10.5424/sjar/2012103-670-11

Scheffler TL, Park S, Gerrard DE (2011). Lessons to learn about postmortem metabolism using the AMPK $3^{R 200 Q}$ mutation in the pig. Meat Science 89: 244-250. https://doi.org/10.1016/j.meatsci. 2011.04.030

SEOC (2013). Informe de la Sociedad Española de Ovinotecnia y Caprinotecnia (SEOC) del sector ovino y caprino en España: Año 2012. Sociedad Española de Ovinotecnia y Caprinotecnia, Madrid, España. 249 pp.

Smulders F, Marsh B, Swartz D, Russell R, Hoenecke $M$ (1990). Beef tenderness and sarcomere length. Meat Science 28: 349-363. https://doi. org/10.1016/0309-1740(90)90048-B

Thompson J (2002). Managing meat tenderness. Meat Science 62: 295-308. https://doi.org/10. 1016/S0309-1740(02)00126-2

Tsiplakou E, Papadomichelakis G, Sparaggis D, Sotirakoglou K, Georgiadou M, Zervas G (2016). The effect of maternal or artificial milk, age and sex on three muscles fatty acid profile of Damascus breed goat kids. Livestock Science 188: 142-152. https://doi.org/10.1016/j.livsci.2016.04.014 
Van Laack R, Kauffman R, Greaser M (2001). Determinants of ultimate $\mathrm{pH}$ of meat. 47 International Congress of Meat Science and Technology, 26-31 agosto, Kraków, Poland, pp. 22-27.

Warris $P$ (2000). Meat science. An introductory text. CABI Publishing, Nueva York, EEUU. 310 pp.

Webb EC, Casey NH, Simela L (2005). Goat meat quality. Small Ruminant Research 60: 153-166. https://doi.org/10.1016/j.smallrumres.2005. 06.009

Wood JD, Richardson RI, Nute GR, Fisher AV, Campo MM, Kasapidou E, Sheard PR, Enser M (2004). Effects of fatty acids on meat quality: a review. Meat Science 66: 21-32. https://doi. org/10.1016/s0309-1740(03)00022-6
Yalcintan H, Akin PD, Ozturk N, Ekiz B, Kocak O, Yilmaz A (2018a). Carcass and meat quality traits of Saanen goat kids reared under natural and artificial systems and slaughtered at different ages. Acta Veterinaria Brno 87: 293-300. https://doi.org/10.2754/avb201887030293

Yalcintan H, Ekiz B, Ozcan M (2018b). Comparison of meat quality characteristics and fatty acid composition of finished goat kids from indigenous and dairy breeds. Tropical Animal Health and Production 50: 1261-1269. https://doi.org/ $10.1007 / \mathrm{s} 11250-018-1553-3$

(Aceptado para publicación el 11 de junio de 2020) 